\title{
Podemos melhorar o olfato na rinite alérgica?
}

\section{Can we improve olfaction in allergic rhinitis?}

Data de receção / Received in: 19/09/2018 Data de aceitação / Accepted for publication in: 28/I I/2018

\author{
Rev Port Imunoalergologia $2019 ; 27$ (2): | $27-134$
}

Marta Martins', Cíntia Cruz', Sofia Farinha', Bárbara Cardoso', Mário Santos², Luís Antunes², Filipe Inácio'

I Serviço de Imunoalergologia, Hospital São Bernardo, Setúbal

2 Serviço de Otorrinolaringologia, Hospital Garcia de Orta, Almada

\section{RESUMO}

Introdução: O olfato pode estar afetado na rinite alérgica (RA). Existem poucos estudos sobre a disfunção olfativa nesta patologia. Realizou-se um estudo piloto com o objetivo de avaliar a função olfativa em doentes com RA antes e depois do tratamento com duas opções farmacológicas diferentes. Métodos: Selecionaram-se aleatoriamente 20 doentes, com RA sem terapêutica em curso, que foram distribuídos por dois grupos: o grupo I fez tratamento com anti-histamínico oral (A-HI) e o grupo 2 com corticoide tópico nasal durante 2 semanas. Os sintomas nasais dos doentes e a sua função olfativa foram avaliados antes e depois da terapêutica através da aplicação do teste de controlo da asma e rinite alérgica (CARAT) e do Barcelona Smell Test (BAST-24). Resultados: Na avaliação pré-terapêutica os dois grupos de doentes apresentaram um score no CARAT inferior a 8 na pontuação das vias aéreas superiores, e na avaliação pós-terapêutica $80 \%$ dos doentes obtiveram um score superior a 8 . Na avaliação do BAST-24, verificou-se globalmente uma melhoria dos resultados após o curso de duas semanas de terapêutica. Na discriminação dos odores, verificou-se no grupo I uma média inicial de 59,5 $\pm 14,42$, que passou a $69 \pm 12,2$ ( $p<0,05$ ); o grupo 2 apresentou

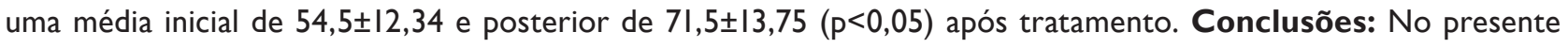
estudo, os pacientes com RA não tratada apresentaram uma melhoria na função olfativa após duas semanas de terapêutica. Nos dois grupos verificou-se uma melhoria estatisticamente significativa no olfato. Não podemos tirar conclusões definitivas, dado o número pequeno da amostra; contudo o corticoide nasal tópico destacou-se a nível da melhoria dos sintomas de rinite e da função olfativa.

Palavras-chave: Rinite alérgica, olfato, anti-histamínico oral, corticoide nasal. 
Marta Martins, Cíntia Cruz, Sofia Farinha, Bárbara Cardoso, Mário Santos, Luís Antunes, Filipe Inácio

\section{ABSTRACT}

Introduction: Smell can be impaired in allergic rhinitis (AR). There are few studies on olfactory dysfunction in this disease. A pilot study was conducted to evaluate olfactory function in patients with allergic rhinitis before and after treatment with two different pharmacological options. Methods: Twenty patients with untreated AR were randomly selected and assigned to 2 groups: group I was treated with oral antihistamine (A-HI) and group 2 with intranasal corticosteroid for 2 weeks. The nasal symptoms of the patients and their olfactory function were evaluated before and after the treatment using the Test of Control of Asthma and Allergic Rhinitis (CARAT) and the Barcelona Smell Test (BAST-24). Results: In the pre-treatment evaluation, the two groups of patients presented a CARAT score of less than 8 in the upper airway score, and in the post-therapy evaluation, $80 \%$ of the patients scored above 8 . In the BAST-24 evaluation an overall improvement was observed after the course of 2 weeks of therapy. In the forced choice of odors, an initial mean of $59.5 \pm 14.42$ was found in group I, which improve to $69 \pm$ 12.2 ( $p<0.05)$; group 2 presented an initial mean of $54.5 \pm 12.34$ and after treatment $71.5 \pm 13.75(p<0.05)$. Conclusions: In our study, patients with untreated $A R$ had an improvement in olfactory function after 2 weeks of therapy. In both groups there was a statistically significant improvement in the sense of smell. We cannot draw definitive conclusions given the small number of the sample, however the topical nasal corticosteroid showed a greater improvement in the symptoms of rhinitis and in the olfactory function.

Keywords: Allergic rhinitis, olfaction, oral antihistamine, intranasal corticosteroid.

\section{INTRODUÇÃO}

A rinite alérgica $(R A)$ é uma das doenças crónicas mais comuns e tem uma prevalência mundial estimada entre $9 \%$ e $42 \%$. Em Portugal a prevalência estimada da RA é de $26.1 \%{ }^{2}$. Esta patologia está associada a comorbidades significativas e a importantes custos de saúde, estando identificado como uma das principais razões para o recurso aos cuidados de saúde primários ${ }^{3}$. A RA tem ainda um impacto negativo nas atividades de vida diária, qualidade do sono e produtividade laboral, pelo que acrescem os custos económicos e sociais associados ${ }^{4}$.

A RA resulta da inflamação da mucosa nasal mediada por lgE após a sensibilização e exposição a alergénios. Clinicamente os sintomas de RA incluem rinorreia aquosa, espirros, congestão e prurido nasal. Outro sintoma importante que está muitas vezes presente na RA é a diminuição da função olfativa ${ }^{5}$. Estudos anteriores que avaliaram com instrumentos quantitativos o sentido do olfato em doentes com RA relataram uma frequência de disfunção olfativa entre $23 \%$ a $80 \%$,7,8. A patogénese da disfunção olfativa na RA parece estar associada por um lado à obstrução nasal que impede que as partículas de odor atinjam o neuroepitélio olfativo" por outro à inflamação nasal eosinofílica presente nesta patologia alérgica ${ }^{10}$, bem como por perturbação da ação da odorant-binding protein"

Embora a disfunção olfativa seja um sintoma importante na RA, há poucos estudos que avaliam a função do olfato nestes doentes. Para além disso, a maioria dos estudos depende de avaliações qualitativas e autorrelatos em detrimento dos métodos quantitativos ${ }^{12}$. Outro aspeto importante diz respeito ao efeito do tratamento da RA na função olfativa, aspeto pouco abordado na literatura.

O objetivo deste estudo foi avaliar a frequência de disfunção olfativa na RA não tratada e investigar a possí- 
vel melhoria de um curso de tratamento com corticosteroide tópico versus anti-histamínico oral no sentido do olfato desses doentes.

\section{MATERIAL E MÉTODOS}

\section{Desenho do estudo}

Estudo-piloto que incluiu um total de 20 doentes com RA seguidos em consulta de otorrinolaringologia. Os doentes foram selecionados aleatoriamente e a sua idade variou entre os 19 e os 52 anos.

O recrutamento dos doentes baseou-se nos seguintes critérios de inclusão: (i) doentes com mais de 18 anos, (ii) com diagnóstico confirmado de RA, (iii) com sintomas de rinite no ano anterior ou precedentes, (iiii) com um teste prick positivo e / ou IgE específica para um aeroalergénio clinicamente relevante; (iiiii) doentes sem tratamento para a RA durante pelo menos duas semanas antes da inclusão no estudo.

Os dados recolhidos foram anonimizados pelos investigadores para a análise. Todos os participantes deram consentimento informado para a sua participação no estudo.

\section{População}

Obtiveram-se os dados demográficos, perfil de sensibilização alergénica e história clínica através de uma análise retrospetiva dos arquivos clínicos e complementados com entrevistas aos doentes. A duração e gravidade da RA foram classificados de acordo com as diretrizes da $A R I A^{13}$.

A presença de comorbidades (asma, dermatite atópica, rinossinusite, otite média, hipertrofia adenoide) também foi avaliada. Doentes com outras doenças crónicas, como fibrose quística, polipose nasal e bronquiectasias, foram excluídos deste estudo, bem como os doentes que tomaram medicação anti-histamínica ou corticoide nas duas semanas anteriores à primeira avaliação deste estudo.

\section{Intervenções do estudo}

\section{Questionário CARAT}

O CARAT é um questionário de auto-preenchimento que avalia o controlo da RA e asma nas últimas 4 semanas; é constituído por 10 questões divididas em dois scores: o score VAS, constituído por 4 questões relativas a sintomas das vias aéreas superiores, e o score VAl, incluindo 6 questões relacionadas com sintomas das vias aéreas inferiores. Foi avaliado o número de respostas "sim" relativo ao score VAS, antes e após 2 semanas de tratamento para a RA.

\section{Barcelona Smell Test}

Foi utilizado o Barcelona Smell Test (BAST-24) ${ }^{14}$ para avaliar o olfato de todos os doentes, antes e após 2 semanas de tratamento para a RA.

O BAST-24 foi realizado numa sala silenciosa, isolada de ruído e bem ventilada, com temperatura controlada $\left(2 \mathrm{I}-23^{\circ} \mathrm{C}\right)$. Os doentes foram vendados e avaliados para 20 odores para o primeiro nervo craniano. Todos os odores estavam em frascos de vidro herméticos. Uma vez abertos, os frascos foram apresentados a $1 \mathrm{~cm}$ das narinas. Os doentes foram avaliados para diferentes características do odor: deteção, identificação e discriminação (4 opções).

Os doentes foram então aleatoriamente distribuídos para um curso de tratamento com anti-histamínico oral ou corticoide tópico, com 10 doentes em cada braço do estudo. Os selecionados para o grupo anti-histamínico (grupo I) tomaram $5 \mathrm{mg}$ de desloratadina uma vez por dia durante 2 semanas e os selecionados para o grupo tópico de corticoide (grupo 2) fizeram spray nasal de propionato de fluticasona $50 \mathrm{mcg}$ I pulverização em cada narina duas vezes por dia durante 2 semanas.

\section{Análise estatística}

Foi feita uma análise descritiva da população estudada em termos das suas características demográficas e distribuição dos doentes segundo as classificações da 
Marta Martins, Cíntia Cruz, Sofia Farinha, Bárbara Cardoso, Mário Santos, Luís Antunes, Filipe Inácio

rinite da ARIA. A análise estatística incluiu a comparação entre controlo da rinite (avaliado pelo CARAT) pré e pós-terapêutica e a comparação da função olfativa (nas diferentes tarefas avaliadas) pré e pós-terapêutica (A-HI versus corticoide tópico nasal). $A$ análise estatística foi realizada com o Medcalc versão I5.I. As comparações foram realizadas com recurso aos testes não paramétricos apropriados em cada caso (Wilcoxon e Man-Whitney). Foi considerado o valor $p<0,05$ como estatisticamente significativo.

\section{RESULTADOS}

\section{Dados demográficos}

Um total de 20 doentes (12 mulheres e 8 homens) foram incluídos no estudo. Os doentes tinham uma média de idades de 33 anos (min 19; máx 52; DP 9,8). A

Quadro I. Características demográficas

\begin{tabular}{|c|c|}
\hline Características e clínica dos doentes & Valores \\
\hline Idade, anos (média $\pm D P)$ & $33,25 \pm 9,84$ \\
\hline Sexo $(F, \%)$ & $12(60 \%)$ \\
\hline Anos desde diagnóstico de rinite (média $\pm D P$ ) & $3,5 \pm 2,44$ \\
\hline $\begin{array}{l}\text { RA intermitente \% } \\
\text { Ligeira } \\
\text { Moderada-grave } \\
\text { RA persistente \% } \\
\text { Ligeira } \\
\text { Moderada-grave }\end{array}$ & $\begin{array}{c}25 \\
5 \\
20 \\
75 \\
5 \\
70\end{array}$ \\
\hline $\begin{array}{l}\text { Comorbilidades } \mathbf{( N )} \\
\text { Asma } \\
\text { Dermatite atópica } \\
\text { Rinossinusite } \\
\text { OM seromucosa } \\
\text { Hipertrofia adenoides }\end{array}$ & $\begin{array}{l}0 \\
1 \\
5 \\
2 \\
5\end{array}$ \\
\hline $\begin{array}{l}\text { Positividade teste prick (N) } \\
\text { Pólenes } \\
\text { Ácaros } \\
\text { Fungos } \\
\text { Faneras } \\
\text { Monossensibilizados } \\
\text { Polissensibilizados }\end{array}$ & $\begin{array}{c}11 \\
13 \\
1 \\
1 \\
7 \\
13\end{array}$ \\
\hline
\end{tabular}

maioria apresentava RA persistente $(75 \%, n=15)$, tendo os restantes 5 doentes RA intermitente (25\%). Quanto à gravidade da RA, verificou-se que $70 \%$ dos doentes apresentava uma RA moderada a grave e $30 \%$ ligeira (Quadro I). Os doentes foram randomizados por 2 grupos: grupo I (tratado com desloratadina, $5 \mathrm{mg}$ ) constituído por 10 doentes ( 6 mulheres, 4 homens) com média de idades de 32,8 \pm II,8 anos (min 19, máx 52) e grupo 2 constituído por 10 doentes $(6$ mulheres e 4 homens) com média de idades de 33,7 \pm 8,06 anos ( $\mathrm{min}$ 24, máx 49).

Em termos de comorbilidades, 5 doentes apresentavam rinossinusite, 5 hipertrofia dos adenóides, 2 otite média seromucosa (OSM) e apenas um doente com dermatite atópica.

\section{Avaliação alergológica}

Todos os doentes apresentaram testes cutâneos positivos, sendo que a maioria apresentava positividade a ácaros $(n=13)$, seguido dos pólenes $(n=\mid I)$. A maioria dos doentes estava polissensibilizada a ácaros e pólenes $(n=13)$.

\section{Sintomas nasais}

Todos os doentes apresentaram no CARAT uma pontuação inferior a 8 no score das vias aéreas, revelando um não controlo da sua RA (Figura I). O CARAT aplicado após terapêutica revelou em $80 \%$ dos doentes resultados superiores a 8 , o que corresponde a um controlo da doença. No grupo I verificou-se que $60 \%$ dos doentes após terapêutica apresentaram valores superiores a 8 e $40 \%$ inferiores ou iguais a 8 (Figura 2). No grupo de tratamento com corticoide nasal tópico verificou-se uma melhoria dos sintomas nasais para $100 \%$ dos doentes, pelo que todos apresentaram resultados superiores a 8 (Figura 3).

\section{Função olfativa}

Os doentes com RA não tratada apresentaram globalmente uma melhoria na função olfativa (na deteção e 


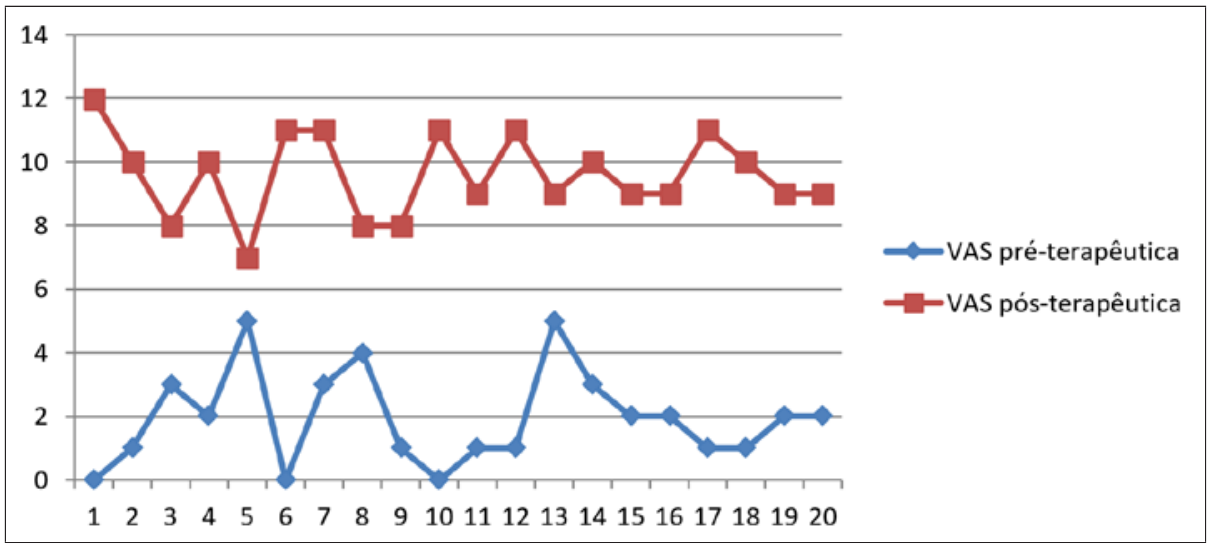

Figura I. Resultados CARAT score VAS pré-terapêutica e pós-terapêutica

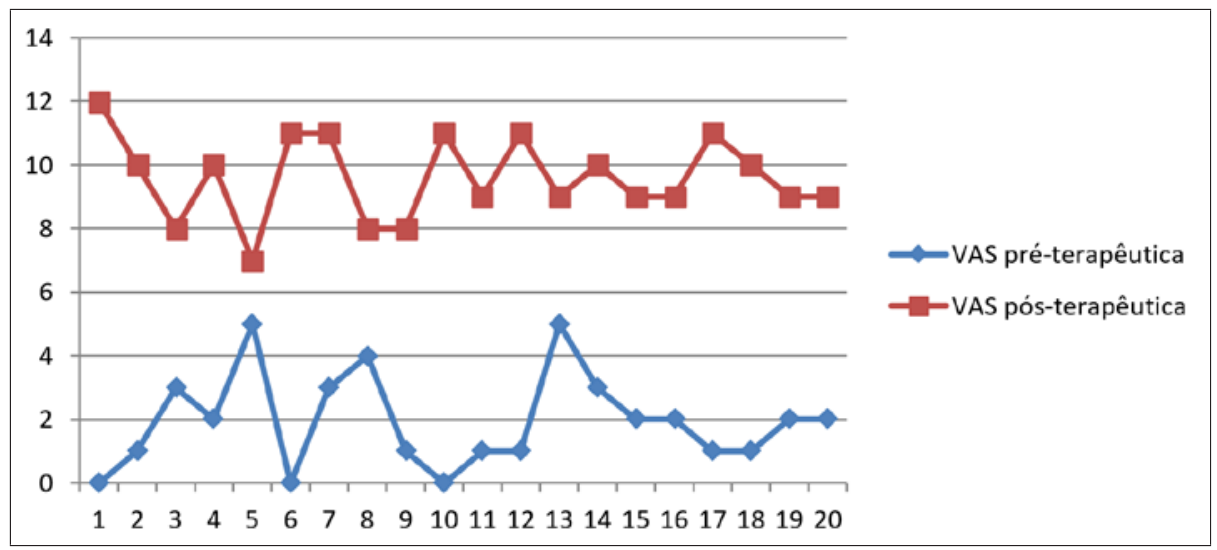

Figura 2. Resultados CARAT score VAS pré-terapêutica e pós-terapêutica no grupo I

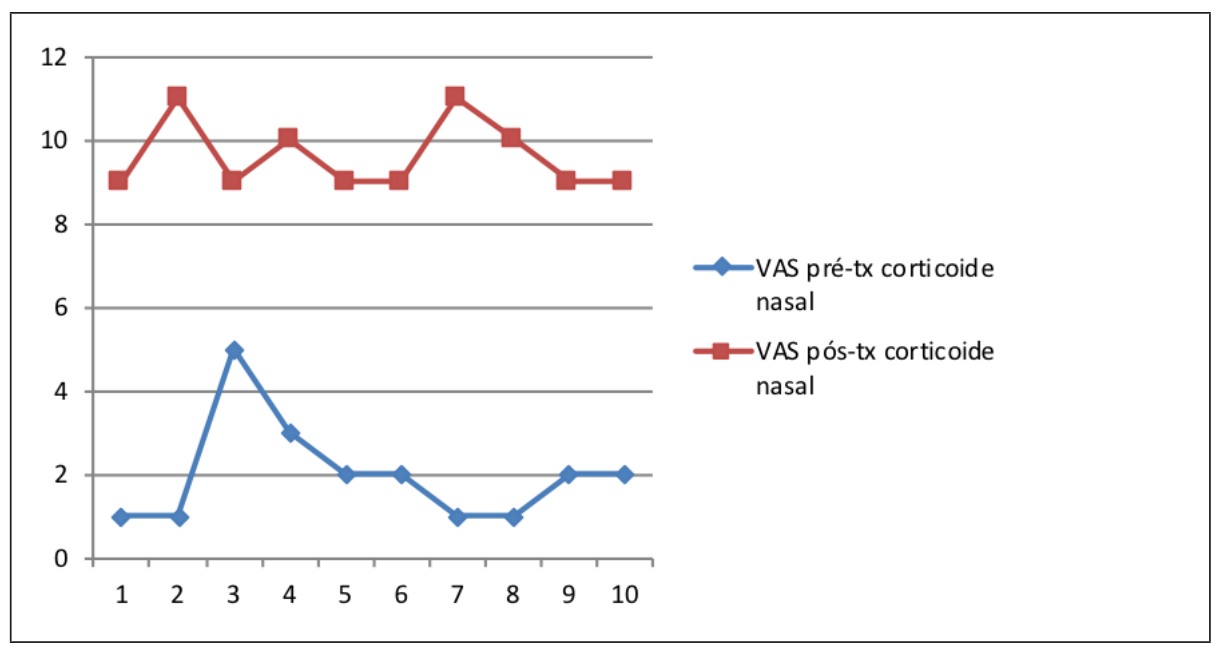

Figura 3. Resultados CARAT score VAS pré-terapêutica e pós-terapêutica no grupo 2 
Marta Martins, Cíntia Cruz, Sofia Farinha, Bárbara Cardoso, Mário Santos, Luís Antunes, Filipe Inácio

Quadro 2. Resultados BAST-24

\begin{tabular}{|l|c|c|c|c|}
\hline & \multirow{2}{*}{$\mathbf{N}$} & I. $^{\mathbf{a}}$ avaliação & 2. $^{\text {a }}$ avaliação & \multirow{2}{*}{$\mathbf{~}$} \\
\cline { 3 - 4 } & & $\mathbf{M} \pm \mathbf{D p}$ & $\mathbf{M} \pm \mathbf{D p}$ & \\
\hline Deteção A-HI (\%) & 10 & $98,0 \pm 2$ & $100 \pm 0$ & 0,3 \\
\hline Discriminação A-HI (\%) & 10 & $59.5 \pm 14,42$ & $69 \pm 12,2$ & 0,0044 \\
\hline Deteção corticoide nasal (\%) & 10 & $99 \pm 2,1$ & $100 \pm 0$ & 0,08 \\
\hline Discriminação corticoide nasal (\%) & 10 & $54,5 \pm 12,34$ & $71,5 \pm 13,75$ & 0,0016 \\
\hline
\end{tabular}

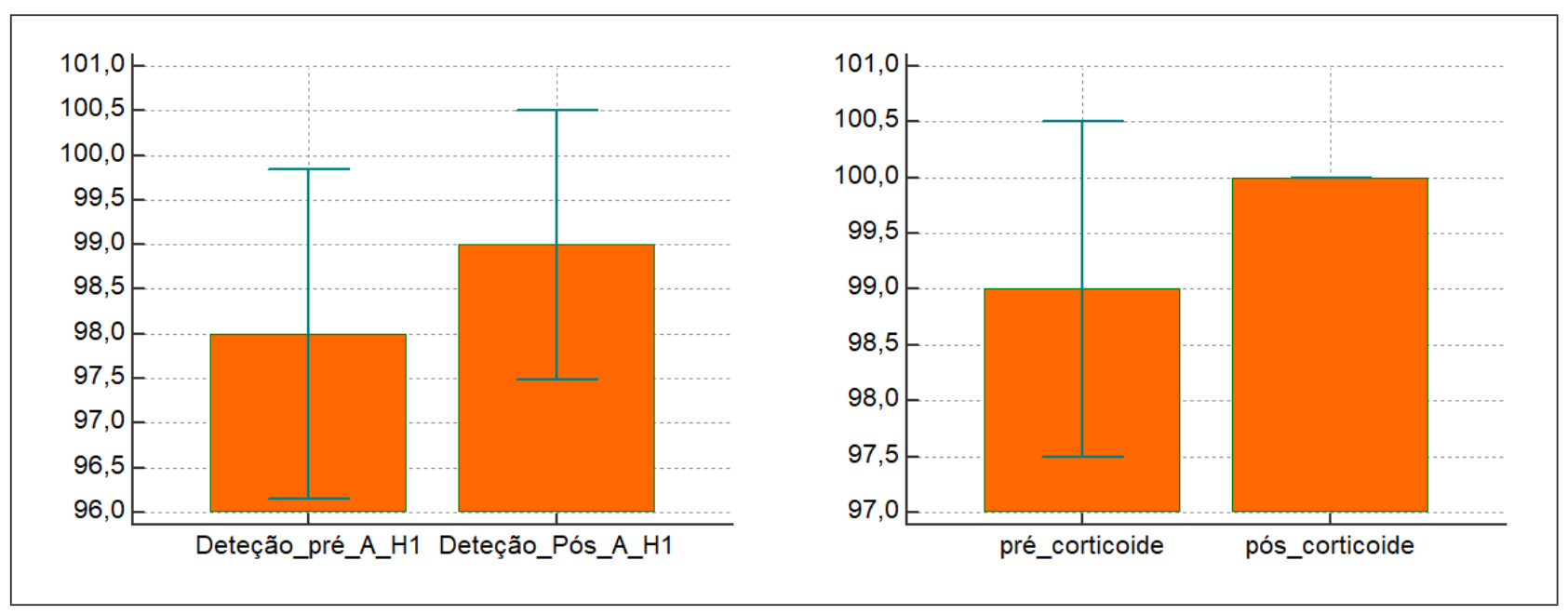

Figura 4. Resultados deteção pré e pós-terapêutica, grupo I (à esquerda) e grupo 2 (à direita)

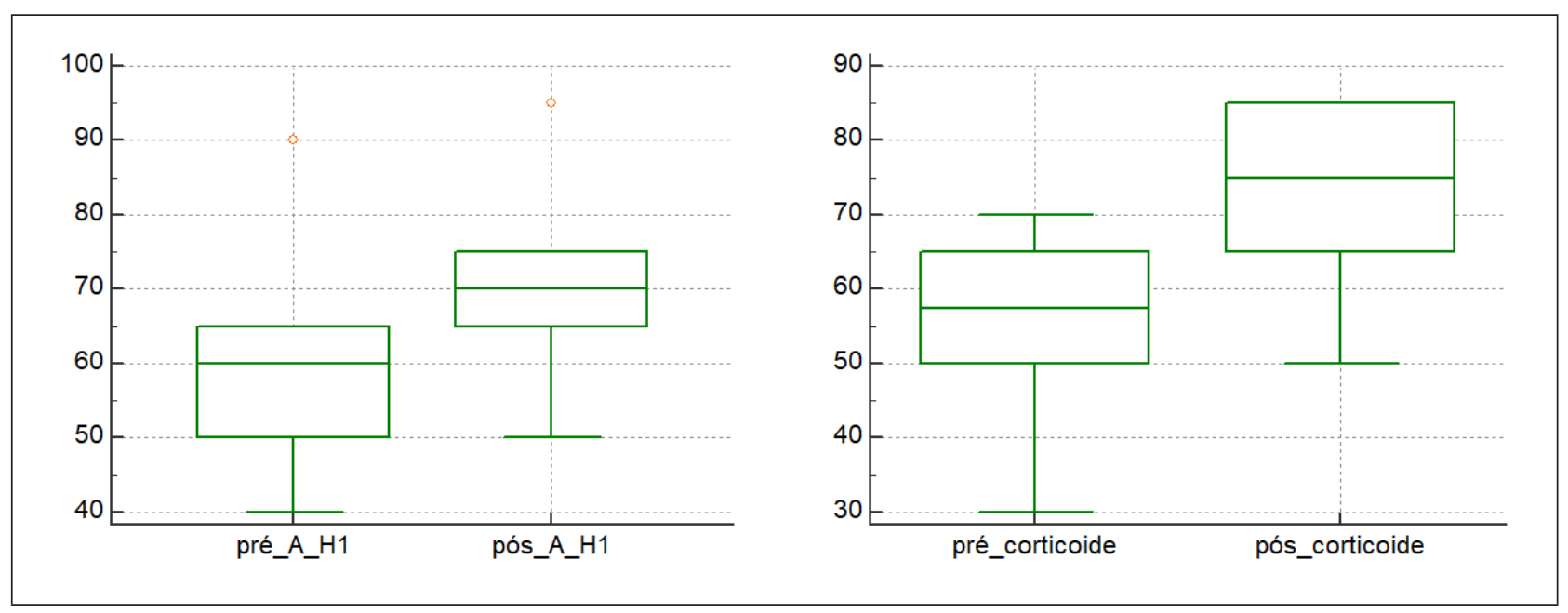

Figura 5. Resultados discriminação pré e pós-terapêutica, grupo I (à esquerda) e grupo 2 (à direita) 
discriminação dos odores) após 2 semanas de terapêutica (Quadro 2).

Nos dois grupos (A-HI versus corticoide tópico nasal) verificou-se uma melhoria estatisticamente significativa na discriminação dos odores (escolha forçada de 4 opções). Ainda que a melhoria na função olfativa tenha sido superior para o grupo que realizou corticoide nasal $(71,5 \pm 13,75 ; p<0,005)$, comparativamente ao grupo que realizou desloratadina $(69 \pm 12,2 ; p<0,005)$, esta diferença não foi estatisticamente significativa.

Três doentes (um no grupo I e dois no grupo 2) não apresentaram alterações nos resultados pós- terapêutica.

\section{DISCUSSÃO}

A RA é uma patologia crónica com uma prevalência elevada em Portugal, sendo responsável por custos diretos e indiretos importantes. Os sintomas de RA têm um impacto na qualidade de vida dos doentes e implicam uma morbilidade associada significativa. A hiposmia é um dos sintomas mais comuns na rinite alérgica, sendo muitas vezes subdiagnosticada e subvalorizada na prática clínica.

Em Portugal não existe um instrumento validado para avaliação do olfato, no entanto o BAST-24 é validado para a população espanhola e utilizado noutros países mediterrâneos, dadas as semelhanças culturais entre estes países, nomeadamente quanto aos odores mais comuns.

Atualmente está a ser desenvolvido e validado um PTSmellTest numa colaboração da Faculdade de Farmácia de Lisboa com o Serviço de ORL do Hospital Garcia de Orta, o que permitirá o seu uso como ferramenta de avaliação objetiva da função olfativa não só na rinite mas também noutras patologias, nomeadamente do foro ORL.

Neste estudo-piloto verificou-se que os doentes com RA não tratada apresentaram uma melhoria na função olfativa após duas semanas de terapêutica. Ainda que a diferença entre as duas opções de tratamento não seja estatisticamente valorizável, verificamos que o grupo tratado com o corticoide nasal tópico apresentou me-
Ihoria dos sintomas de rinite, avaliados pelo CARAT, bem como do sentido do olfato. De facto os corticoides nasais tópicos têm um papel determinante na redução da inflamação local presente na RA e, consequentemente, na melhoria do olfato ${ }^{8}$.

Este estudo apresenta algumas limitações, nomeadamente o pequeno número da amostra. Para além disso, não foi avaliada a possibilidade da terapêutica combinada, prática consensual no tratamento da RA e que provavelmente condiciona uma melhoria mais expressiva ao nível olfativo. Seria ainda interessante avaliar a perceção subjetiva dos doentes relativamente à função olfativa antes e depois do tratamento para a RA, bem como comparar com uma avaliação quantitativa do sentido do olfato.

\section{CONCLUSÕES}

Os resultados deste trabalho mostram uma melhoria da função do olfato após tratamento farmacológico para a RA. Apesar de ambas as opções terapêuticas apresentarem melhoria estatisticamente significativa na função do olfato, verificámos que o spray nasal de propionato de fluticasona destacou-se, quer a nível da melhoria dos sintomas de rinite, quer a nível da função olfativa.

Este estudo permite constatar que o olfato é um sentido afetado na RA, pelo que a sua avaliação por instrumentos qualitativos e/ou quantitativos deve ser considerada na avaliação de um doente com $\mathrm{RA}$.

\section{Conflito de interesses}

Os autores declaram que não existem conflitos de interesse.

\section{Contacto:}

Marta Martins Batista

Serviço de Imunoalergologia

Hospital São Bernardo

R. Camilo Castelo Branco 2910-446 Setúbal

E-mail:marttamarttins@gmail.com 


\section{REFERÊNCIAS}

I. Settipane RA, Charnock DR. Epidemiology of rhinitis: allergic and nonallergic. Clin Allergy Immunol 2007; 19:23-34.

2. Todo-Bom A, Loureiro C, Almeida MM, Nunes C, Delgado L, Castel-Branco G, Bousquet J. Epidemiology of rhinitis in Portugal: evaluation of the intermittent and the persistent types. Allergy 2007;62:1038-43

3. Hayden ML. Allergic rhinitis: a growing primary care challenge. J Am Acad Nurse Pract 200I, 13:545-5I.

4. Crystal-Peters J, Crown W, Goetzel R, Schutt D. The cost of productivity losses associated with allergic rhinitis. Am J Manag Care 2000;6:373-8.

5. Stuck BA, Hummel T. Olfaction in allergic rhinitis: A systematic review. J Allergy Clin Immunol 2015;136:1460-70.

6. Binder E, Holopainen E, Malmberg H, Salo O. Anamnestic data in allergic rhinitis. Allergy 1982;37:389-96.

7. Guss J, Doghramji L, Reger C, Chiu AG. Olfactory dysfunction in allergic rhinitis. ORLJ Otorhinolaryngol Relat Spec 2009;71:268-72.

8. Sivam A, Jeswani S, Reder L, Wang J, Deniteo M, Taxy J, et al. Olfactory cleft inflammation is present in seasonal allergic rhini- tis and is reduced with intranasal steroids. Am J Rhinol Allergy 2010;24:286-90.

9. Doty RL, Frye R. Influence of nasal obstruction on smell function. Otolaryngol Clin North Am 1989;22:397-4II.

10. Klimek L. Eggers G. Olfactory dysfunction in allergic rhinitis is related to nasal eosinophilic inflammation. J Allergy Clin Immunol 1997;100:158-64.

II. Stephen Dd. Roper (2012). Gustatory and olfactory sensory transduction. In: Nicholas Sperelakis (Eds), Cell physiology sourcebook $4^{\text {th }}$ Edition (Chap.39, pp.68I-98). Academic Press Elsevier Inc.

12. Langdon C, Guilemany JM, Valls M, et al. Allergic rhinitis causes loss of smell in children: The OLFAPEDRIAL study. Pediatr Allergy Immunol 2016;27:867-70.

13. Bousquet J, Khaltaev N, Cruz AA, et al. Allergic rhinitis and its impact on asthma (ARIA) 2008 Uptade (in collabration with the World Health Organization, GA2LEN and AllerGen). Allergy 2008: 63 (Suppl.86):8-160.

14. Cardesín A, Alobid I, Benítez P, Sierra E, de Haro J, BernalSprekelsen M, et al. Barcelona Smell Test - 24 (BAST-24): validation and smell characteristics in the healthy Spanish population. Rhinology 2006; 44: 83-9. 